\title{
Efficacy and safety of Infliximab for steroid-resistant immune-related adverse events: A retrospective study
}

\author{
YUKIO KADOKAWA $^{1}$, MARI TAKAGI ${ }^{1}$, TOMOE YOSHIDA ${ }^{1}$, AKITOSHI TATSUMI ${ }^{2}$, KEIKO FUJITA ${ }^{1}$, \\ TAKAKO INOUE ${ }^{3}$, SHUICHI OHE $^{4}$, YASUTOMO NAKAI ${ }^{5}$, SACHIKO YAMAMOTO $^{6}$, TOMOYUKI OTSUKA ${ }^{7}$, \\ RYU ISHIHARA ${ }^{6}$, TAIKI ISEI ${ }^{4}$, TORU KUMAGAI ${ }^{3}$, KAZUO NISHIMURA $^{5}$ and FUMIO IMAMURA ${ }^{7}$ \\ ${ }^{1}$ Department of Pharmacy, Osaka International Cancer Institute, Osaka Prefectural Hospital Organization, Osaka 541-8567; \\ ${ }^{2}$ Education and Research Center for Clinical Pharmacy, Kobe Pharmaceutical University, Kobe, Hyogo 658-8558; \\ Departments of ${ }^{3}$ Respiratory Medicine, ${ }^{4}$ Dermatologic Oncology, ${ }^{5}$ Urology, ${ }^{6}$ Gastroenterology and ${ }^{7}$ Medical Oncology, \\ Osaka International Cancer Institute, Osaka Prefectural Hospital Organization, Osaka 541-8567, Japan
}

Received April 6, 2020; Accepted January 12, 2021

DOI: $10.3892 / \mathrm{mco} .2021 .2227$

\begin{abstract}
The present study investigated outcomes of infliximab (IFX) treatment among 8 Japanese patients with various types of cancer (4 with malignant melanoma, 3 with lung cancer and 1 with renal cancer) who developed severe steroid-resistant immune-related adverse events (irAEs) in association with immune checkpoint inhibitors (ICIs) to determine its efficacy and safety. Information, including patient background, treatment progress, examination data and imaging data, was collected retrospectively from electronic medical records. Adverse reactions were evaluated using the Common Terminology Criteria for Adverse Events version 4.0. Specific ICIs used were anti-PD-1, anti-PD-L1 and anti-CTLA-4 antibody preparations in 7, 2 and 5 patients, respectively. Specific irAEs included grade 3 diarrhea/colitis in 7 patients and disseminated intravascular coagulation and myocarditis attributed to autoimmune activation in 1 patient. The median duration between systemic steroid and IFX treatments was 9 (range, 2-39) days. A total of 3 patients responded to IFX, 1 of whom responded after one dose and 2 responded after two doses. Respective diseases improved to
\end{abstract}

Correspondence to: Mr. Yukio Kadokawa, Department of Pharmacy, Osaka International Cancer Institute, Osaka Prefectural Hospital Organization, 3-1-69 Otemae, Chuo-ku, Osaka 541-8567, Japan

E-mail:kadocchi41@gmail.com

Abbreviations: $\mathrm{CD}$, Clostridium difficile; ICI, immune checkpoint inhibitor; IFX, infliximab; irAEs, immune-related adverse events; CMV, cytomegalovirus; CTLA-4, T-lymphocyte antigen-4; PD-1, programmed cell death-1; PD-L1, programmed cell death-ligand 1; PSL, prednisolone; IPI, ipilimumab; Nivo, nivolumab; CRT, chemoradiation therapy; TNF- $\alpha$, tumor necrosis factor- $\alpha$; CyA, cyclosporine

Key words: infliximab, immune-related adverse event, immune checkpoint inhibitor, diarrhea/colitis, steroid resistance grade 0 after a median of 18 (range, 9-32) days. No AEs were attributable to IFX. Additionally, anti-cytomegalovirus (CMV) and antibacterial agents were administered in parallel given the presence of CMV and Clostridium difficile (CD) infections in all patients, except in 1 exhibiting a marked IFX response after one dose. The combination of highly immunosuppressive IFX and high-dose systemic steroid administration over a long period presumably predisposed the patients to opportunistic enteric infections. Accordingly, early initiation of IFX treatment in conjunction with systemic steroid therapy should be considered for severe diarrhea/colitis and other irAEs. However, the possibility for CMV and CD infections should be recognized, and for these the treatment strategy may need to be modified at an early stage.

\section{Introduction}

Immune checkpoint inhibitors (ICIs) are promising drugs that can potentiate the immune system of cancer patients for disease treatment. ICIs block endogenous factors, such as cytotoxic T-lymphocyte antigen-4 (CTLA-4) and programmed cell death-1 (PD-1), thereby enhancing the antitumor effect. ICIs, such as nivolumab (Nivo), have reportedly extended the patient overall survival in cases of different types of cancer and have been approved in several countries. Meanwhile, Immune-related adverse events (irAEs), which often occur in association with immune checkpoint inhibitor (ICI) treatment, require early detection and appropriate management considering their potentially fatal outcomes. Among irAEs, diarrhea/colitis occurs particularly frequently, and serious complications, such as intestinal perforation, may follow unless timely and appropriate treatment is provided (1). The American Society of Clinical Oncology guideline includes an organ system-based management algorithm showing recommended management procedures for various irAEs according to grade (2). In line with this, steroid therapy with approximately $1 \mathrm{mg} / \mathrm{kg} /$ day prednisolone (PSL) equivalent is immediately initiated for grade 3 diarrhea/colitis. However, when symptoms do not improve, infliximab (IFX) treatment, generally at a dose of $5 \mathrm{mg} / \mathrm{kg} / \mathrm{day}$ according to the 
administration for ulcerative colitis (3-11), has been recommended. Single-dose IFX administration has often been selected as an irAE treatment, with additional doses administered only when no improvements occur after the first dose. Perez-Ruiz et al (12) have demonstrated that the inhibition of tumor necrosis factor- $\alpha$ (TNF- $\alpha$ ) after ICI administration may prevent the occurrence of severe colitis. IFX, approved in 1999 in the United States and in 2003 in Japan, is an anti-TNF- $\alpha$ antibody drug that binds to and neutralizes the action of TNF- $\alpha$, which plays a key role in the development and exacerbation of rheumatoid arthritis. While transient headache and nausea can occur as short-term adverse reactions to IFX, these symptoms are mild. However, it is imperative to pre-emptively identify symptoms of medium- and long-term adverse reactions such as infectious diseases, demyelinating diseases, aplastic anemia, malignant tumors, autoimmune diseases, and heart failure, among which infectious diseases are of particular concern. Several case reports have described successful treatment of steroid-resistant ICI-induced diarrhea/colitis with IFX in patients receiving ipilimumab (IPI), an anti-cytotoxic T-lymphocyte antigen-4 (CTLA-4) antibody preparation (3-6), as well as anti-programmed cell death-1 (PD-1) antibody or anti-programmed cell death-ligand 1 (PD-L1) antibody preparations (7-11). However, we were unable to identify any report systematically dealing with the required number of IFX doses or the time to switch to after treatment.

Therefore, this study investigated the outcomes of IFX treatment among Japanese cancer patients who developed severe steroid-resistant irAEs induced by various ICIs and examined its efficacy and safety for severe steroid-resistant irAEs.

\section{Materials and methods}

Patients. Among nine Japanese patients with cancer who underwent IFX treatment for ICI-induced steroid-resistant irAEs at the Osaka International Cancer Institute (hereinafter referred to as the 'Institution') between January 2018 and June 2019, eight whose electronic medical records contained the necessary treatment information were included; one patient who participated in a clinical trial was excluded.

We obtained 'consent to the publication of a paper related to the course of irAE treatment' from a patient who was alive and able to undergo follow-up examinations (case no. 2). For the patients who could not provide consent, regardless of whether they were deceased or owing to other reasons, we used the opt-out submitted when we requested an approval from the Institutional Review Board as a substitute for the consent.

Information collected. Electronic medical records were retrospectively investigated to collect information, such as patient background, treatment progress, examination data, and imaging data. AEs were assessed using the Common Terminology Criteria for Adverse Events version 4.0. The first of 7 consecutive days during which diarrhea severity remained grade 0 was defined as the day of resolution.

Statistical analysis. In this study, we used the statistical software Microsoft Excel 2013 to calculate only the median.

\section{Results}

Background and irAE treatment details. Patient background and irAE treatment details are summarized in Table I. Six male and two female patients, with a median (range) age and body weight of 66 (58-74) years and 60.0 (38.0-85.1) kg, respectively, were included in this study. Four patients presented with malignant melanoma, three with lung cancer, and one with kidney cancer. Specific ICIs used included anti-PD-1, anti-PD-L1, and anti-CTLA-4 antibody preparations in seven, two, and five patients, respectively.

irAE treatment was switched from steroids to IFX since systemic steroid treatment was ineffective for grade $\geq 3$ diarrhea/colitis (case nos. 1-7) or for disseminated intravascular coagulation and myocarditis resulting from activated autoimmunity (case no. 8). Grade 3 diarrhea/colitis occurred at a median (range) of 119 (5-1,011) days after the first ICI dose or at a median (range) of 15.5 (5-75) days after the last ICI dose. Grade 3 hepatitis preceded diarrhea/colitis in one case (case no. 4).

Three patients who responded to IFX satisfied the defined resolution (case nos. 1, 5 and 8). Accordingly, responses to IFX occurred after one dose in case no. 1 and after two doses in case nos. 5 and 8 (in case no. 5, an accessory third dose was administered, but the defined resolution was satisfied after two IFX doses). Although the treatment was transiently effective in one case (case no. 2), irAEs relapsed. Two patients did not respond to IFX (case nos. 3 and 4), while two patients (case nos. 6 and 7) underwent rapid deterioration of their general condition after IFX treatment initiation and died before the IFX effect was noted. The cause of death was irAE diarrhea and multi-organ failure in case no. 6 and respiratory failure due to multiple lung metastases and pulmonary congestion in case no. 7. No causal relationships were observed between IFX treatment and deaths. The median (range) number of days between systemic steroid treatment and IFX initiation was 9 (2-39) days. In three IFX responders, resolution to grade 0 required a median (range) of 18 (9-32) days after IFX treatment initiation. No AEs were attributable to IFX. No patients developed cytomegalovirus (CMV) or Clostridium difficile (CD) infection when grade 3 irAEs occurred.

Among four patients who experienced the relapse of grade 3 diarrhea/colitis after IFX administration or were refractory to IFX, three (case nos. 2, 4 and 5) developed CMV infection and one (case no. 3) developed CD infection after IFX initiation. Detailed treatment courses are described in separate sections for case no. 1 , in whom IFX was effective, and case no. 2 , in whom irAE relapsed repeatedly.

Since different hormone level between women in menstruation period and women without menstruation period would impact the resistance to steroid, case reports did not list premenopausal women.

Case reports

Case no. 1. The case was a 44-year-old man with a body weight of $69.8 \mathrm{~kg}$.

Diseases: Malignant melanoma of the head, with left cervical lymph node, multiple cutaneous/subcutaneous, lung, and multiple brain metastases (stage IV) 


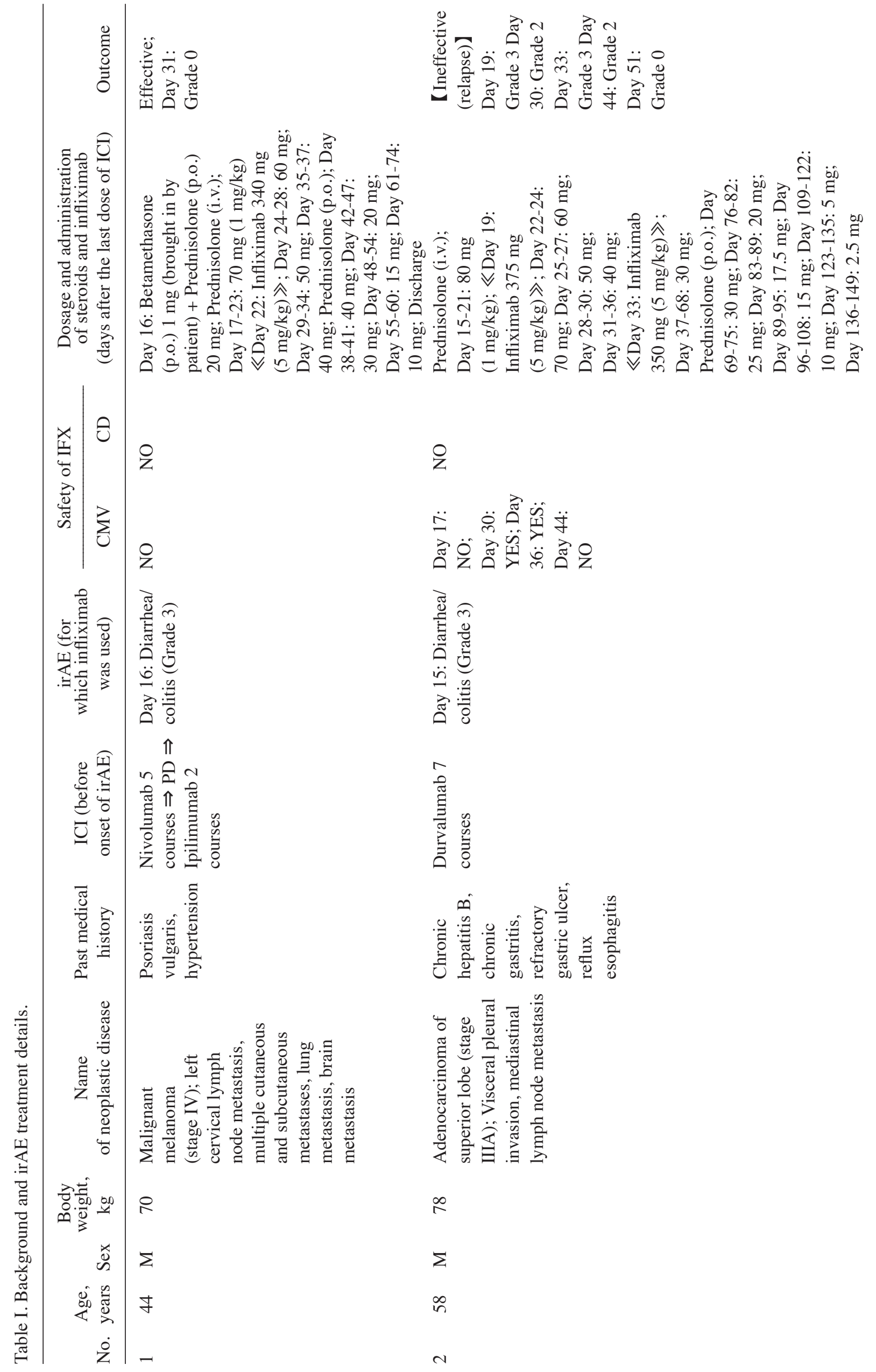




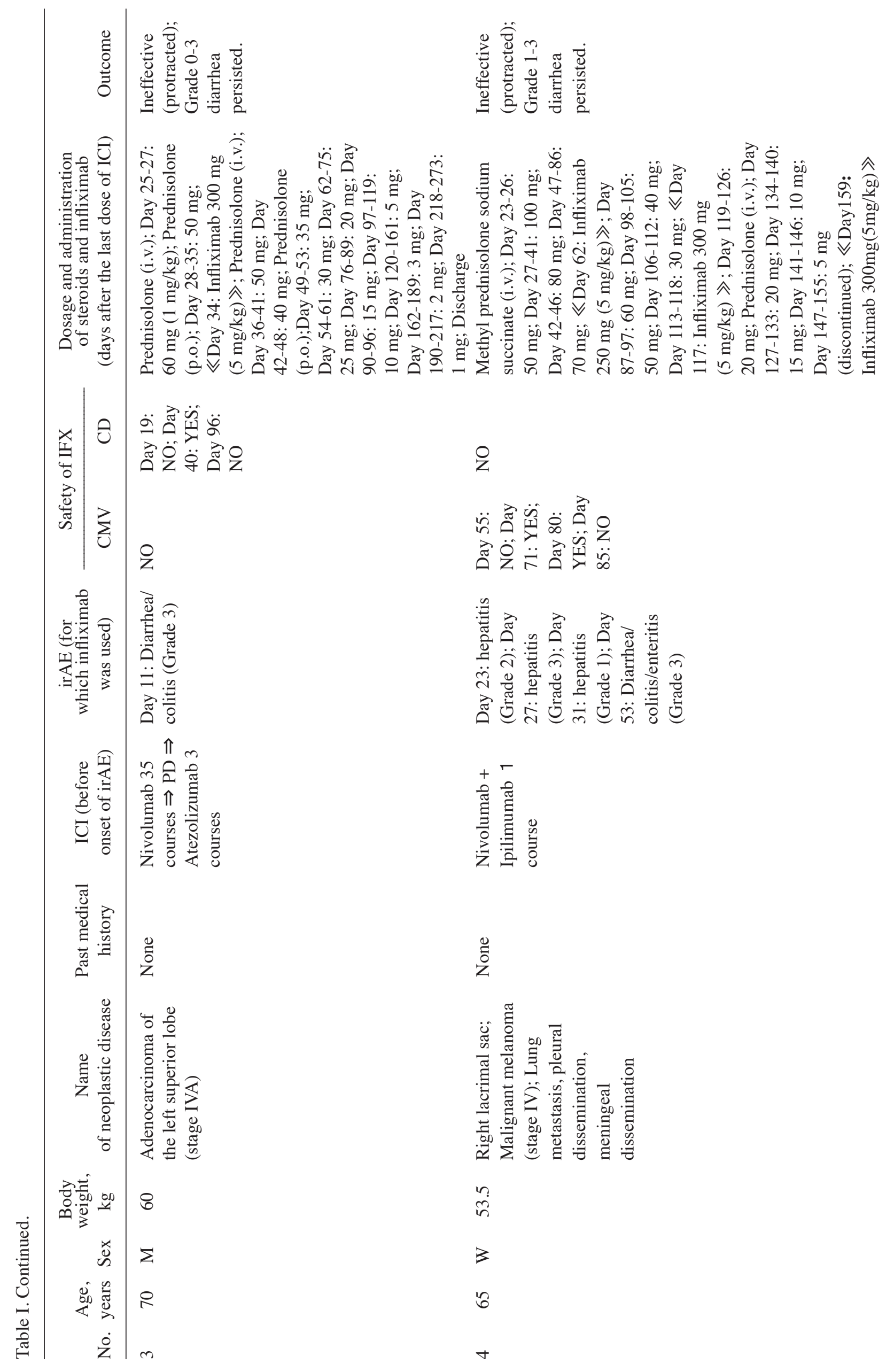




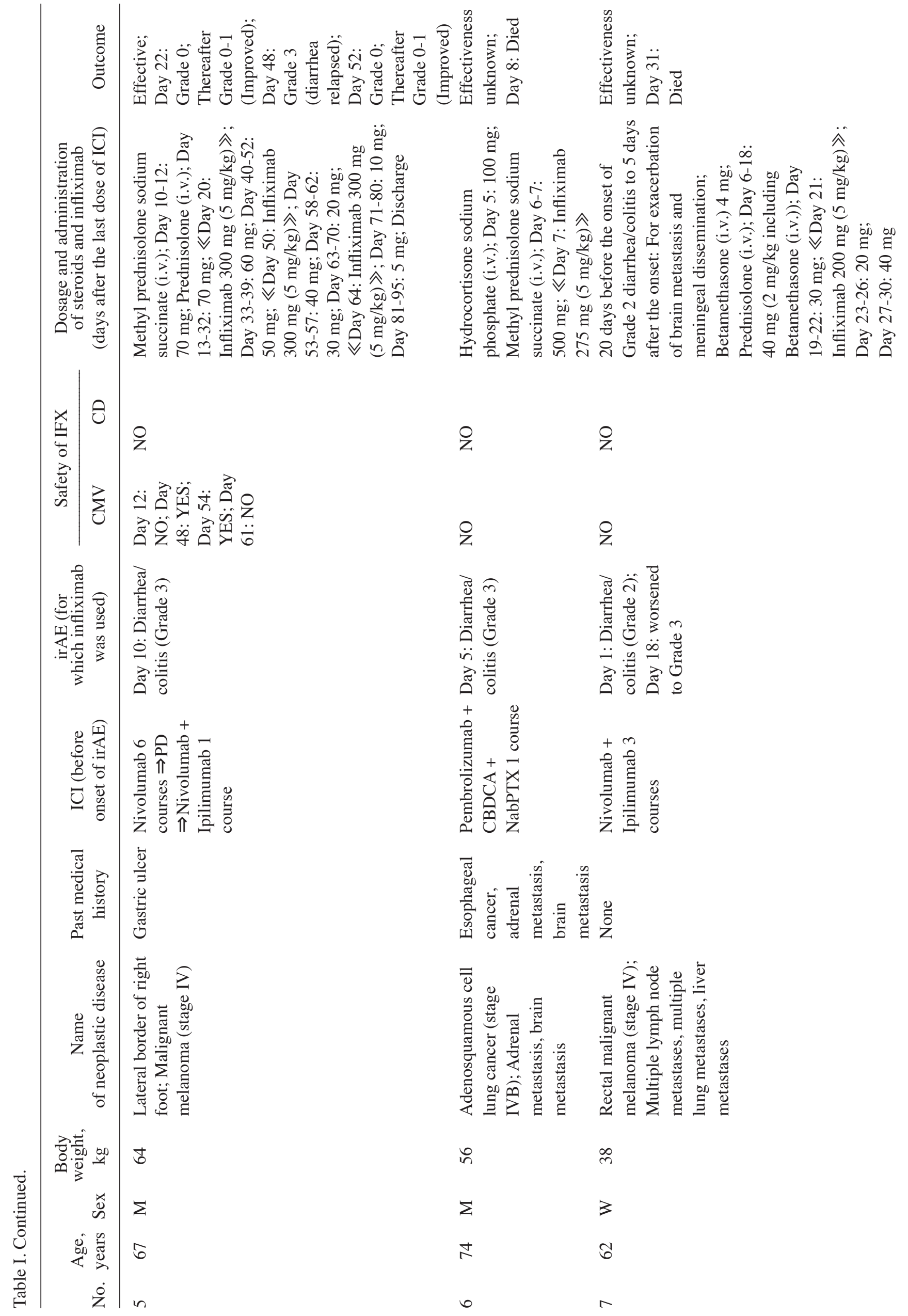




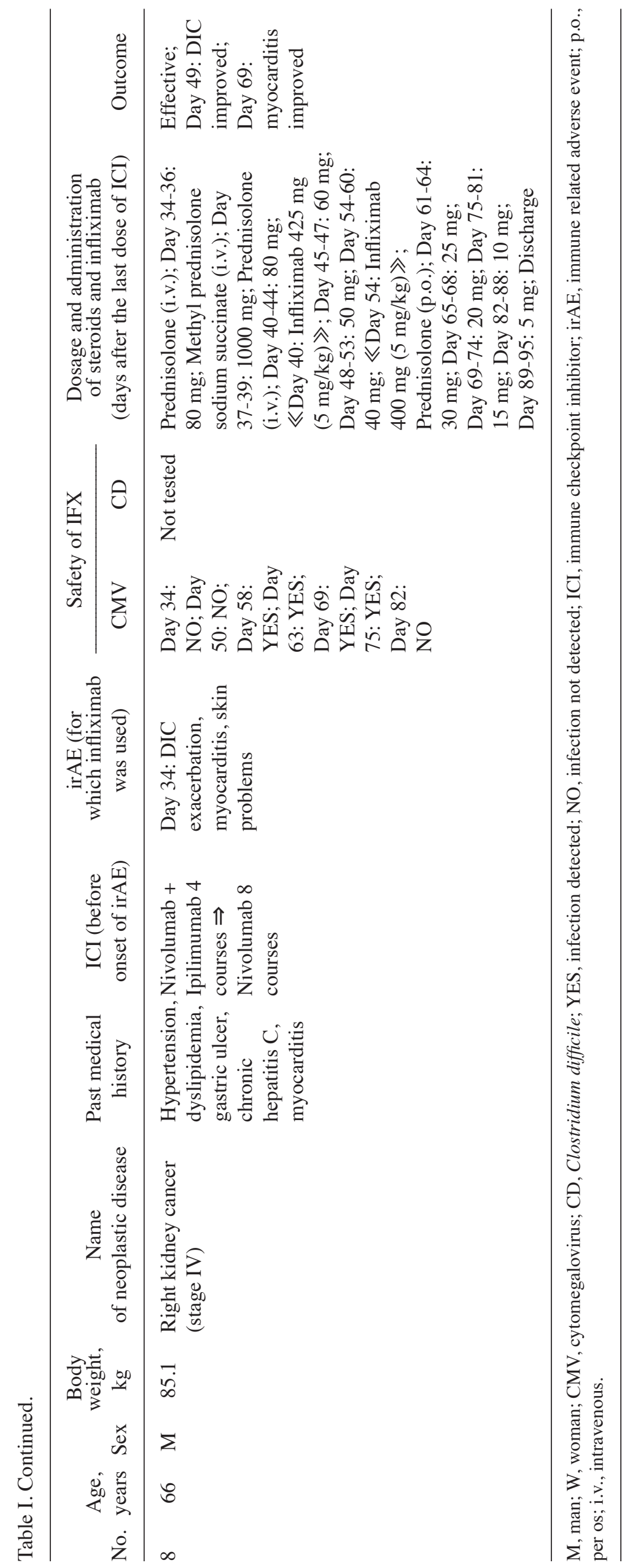



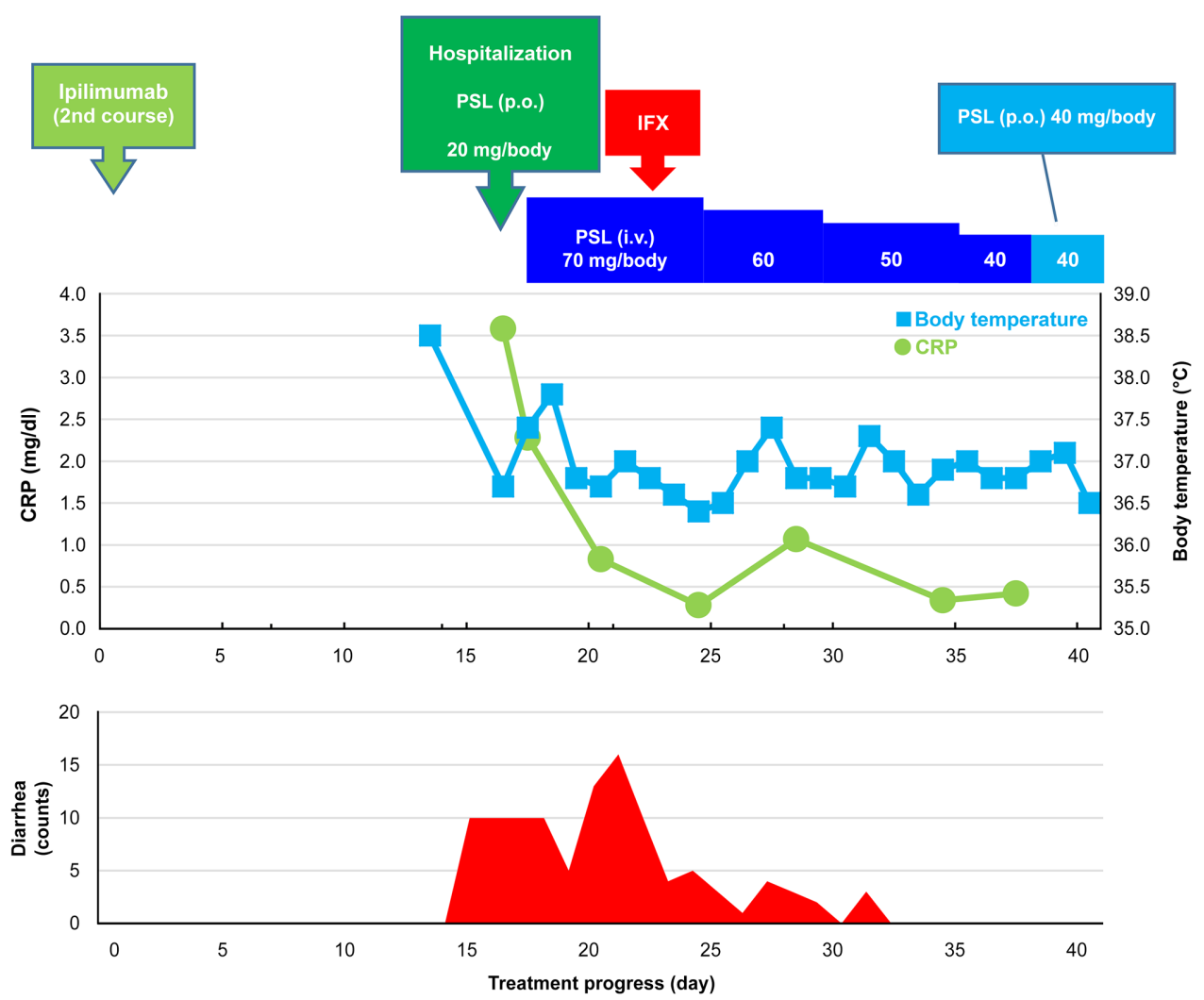

Figure 1. Clinical course after two courses of ipilimumab in case no. 1. IFX, infliximab; PSL, prednisolone; CRP, C-reactive protein; i.v., intravenous; p.o., per os.

A

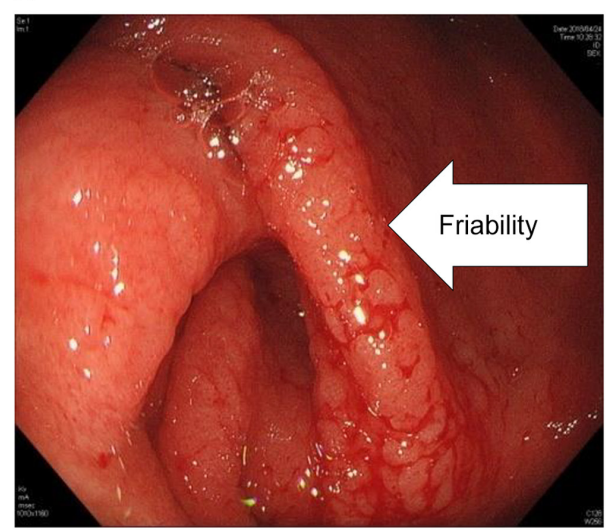

Figure 2. Lower gastrointestinal endoscopy in case no. 1 (day 16). (A) Mucosal friability was noted. (B) Erosion was noted.

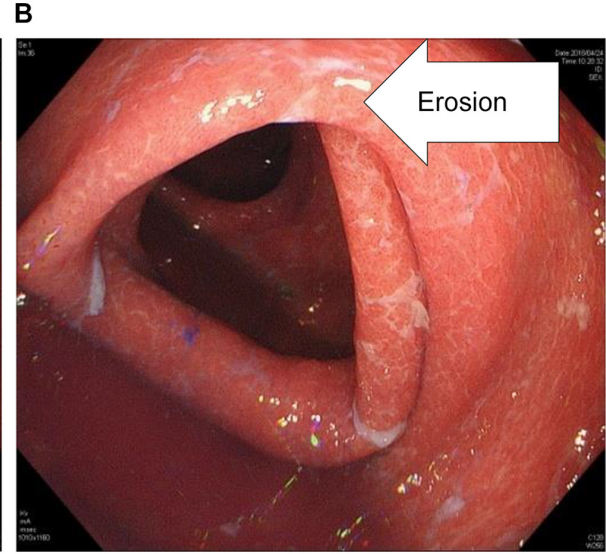

Past medical history: Psoriasis vulgaris and hypertension

History of the present illness: The patient underwent extended resection and dissection of the left cervical lymph node metastases in June 2017. Multiple cutaneous metastases were noted in November 2017, for which extended resection, flap surgery, and resection of a subcutaneous mass in his left back were performed. Although five courses of nivolumab (Nivo) monotherapy had been administered since December 2017, the disease still progressed. Therefore, the treatment was switched to IPI monotherapy as the second-line treatment in February 2018.

Treatment course: The clinical course after two courses of IPI is presented in Fig. 1. The day on which the second IPI course was introduced was set as day 0 . The patient was febrile $\left(38.5^{\circ} \mathrm{C}\right)$ on day 13 , and grade 3 colitis/diarrhea occurred on day 15 . The patient was subsequently hospitalized on day 16 due to diarrhea occurring 10 times/day (grade 3). Endoscopy findings revealed mucosal friability and erosion in the lower gastrointestinal tract (Fig. 2). Laboratory findings on admission are summarized in Table II. The patient was instructed to fast, and replacement fluid and PSL $20 \mathrm{mg} /$ body per os were initiated. Although the PSL dose was increased to $1 \mathrm{mg} / \mathrm{kg} /$ day ( $70 \mathrm{mg} /$ body/day) on day 17, no improvements were noted even after several days; thus, a diagnosis of steroid-resistant colitis/diarrhea was established. Accordingly, IFX $5 \mathrm{mg} / \mathrm{kg} /$ day (340 mg/body/day) was administered intravenously on the seventh hospital day (day 22). The number of bowel movements started decreasing a day after IFX administration (day 23), 
Table II. Case no. 1: Laboratory findings on admission.

\begin{tabular}{|c|c|}
\hline Variable & Value \\
\hline \multicolumn{2}{|l|}{ Hematology } \\
\hline WBC & $6.56 \times 10^{3} / \mu 1$ \\
\hline Neutro & $65.9 \%$ \\
\hline Lympho & $15.8 \%$ \\
\hline Mono & $10.6 \%$ \\
\hline Eosino & $3.1 \%$ \\
\hline Baso & $1.0 \%$ \\
\hline $\mathrm{RBC}$ & $4.96 \times 10^{4} / \mu 1$ \\
\hline $\mathrm{Hb}$ & $14.1 \mathrm{~g} / \mathrm{dl}$ \\
\hline $\mathrm{Ht}$ & $42.5 \%$ \\
\hline PLT & $28.3 \times 10^{4} / \mu 1$ \\
\hline CMV antigen & $(-)$ \\
\hline Fecal culture & $(-)$ \\
\hline Fecal CD toxin & $(-)$ \\
\hline \multicolumn{2}{|l|}{ Biochemistry } \\
\hline Alb & $3.4 \mathrm{~g} / \mathrm{dl}$ \\
\hline AST & $41 \mathrm{U} / 1$ \\
\hline ALT & $30 \mathrm{U} / 1$ \\
\hline LDH & $3,119 \mathrm{U} / 1$ \\
\hline ALP & $219 \mathrm{U} / 1$ \\
\hline$\gamma$-GTP & $60 \mathrm{U} / 1$ \\
\hline CK & $82 \mathrm{U} / 1$ \\
\hline $\mathrm{Cr}$ & $1.11 \mathrm{mg} / \mathrm{dl}$ \\
\hline BUN & 13 mg/dl \\
\hline CRP & $3.58 \mathrm{mg} / \mathrm{dl}$ \\
\hline $\mathrm{Na}$ & $135 \mathrm{mmol} / \mathrm{l}$ \\
\hline $\mathrm{K}$ & $4.2 \mathrm{mmol} / \mathrm{l}$ \\
\hline $\mathrm{Cl}$ & $99 \mathrm{mmol} / \mathrm{l}$ \\
\hline TSH & $0.15 \mu \mathrm{U} / \mathrm{ml}$ \\
\hline FT4 & $0.9 \mathrm{ng} / \mathrm{dl}$ \\
\hline
\end{tabular}

WBC, white blood cell; Neutro, neutrophils; Lympho, lymphocyte; Mono, monocyte; Eosino, eosinophils; Baso, basophil; RBC, red blood cell; Hb, hemoglobin; Ht, hematocrit; PLT, platelet; Alb, serum albumin; AST, aspartate aminotransferase; ALT, alanine aminotransferase; LDH, lactate dehydrogenase; ALP, alkaline phosphatase; $\gamma$-GTP, $\gamma$-glutamyl transpeptidase; CK, creatine kinase; $\mathrm{Cr}$, creatinine clearance; BUN, blood urea nitrogen; $\mathrm{Na}$, serum sodium; $\mathrm{K}$, serum potassium; $\mathrm{Cl}$, serum chlorine; TSH, thyroid stimulating hormone; FT4, free thyroxine 4; CMV, cytomegalovirus; CD, Clostridium difficile.

and stool passage normalized 9 days after IFX administration (day 31). The patient was subsequently discharged on day 45.

Case no. 2. The case was a 58-year-old man with a body weight of $78.3 \mathrm{~kg}$.

Diseases: Adenocarcinoma of the right superior lobe, with visceral pleural invasion and mediastinal lymph node metastasis (stage IIIA: T2aN2M0).

Past medical history: Chronic hepatitis B, chronic gastritis, refractory gastric ulcer, and reflux esophagitis.
Table III. Case no. 2: Laboratory findings on admission.

\begin{tabular}{|c|c|}
\hline Variable & Value \\
\hline \multicolumn{2}{|l|}{ Hematology } \\
\hline WBC & $8.69 \times 10^{3} / \mu 1$ \\
\hline Neutro & $75.3 \%$ \\
\hline Lympho & $12.5 \%$ \\
\hline Mono & $5.7 \%$ \\
\hline Eosino & $3.9 \%$ \\
\hline Baso & $0.3 \%$ \\
\hline $\mathrm{RBC}$ & $4.58 \times 10^{4} / \mu 1$ \\
\hline $\mathrm{Hb}$ & $12.5 \mathrm{~g} / \mathrm{dl}$ \\
\hline $\mathrm{Ht}$ & $40.2 \%$ \\
\hline PLT & $33.4 \times 10^{4} / \mu 1$ \\
\hline CMV antigen & $(-)$ \\
\hline Fecal culture & $(-)$ \\
\hline Fecal CD toxin & $(-)$ \\
\hline \multicolumn{2}{|l|}{ Biochemistry } \\
\hline $\mathrm{TP}$ & $6.8 \mathrm{~g} / \mathrm{dl}$ \\
\hline Alb & $3.4 \mathrm{~g} / \mathrm{dl}$ \\
\hline AST & $14 \mathrm{U} / 1$ \\
\hline ALT & $13 \mathrm{U} / 1$ \\
\hline $\mathrm{LDH}$ & $199 \mathrm{U} / 1$ \\
\hline$\gamma$-GTP & $23 \mathrm{U} / 1$ \\
\hline $\mathrm{Cr}$ & $0.85 \mathrm{mg} / \mathrm{dl}$ \\
\hline CRP & $6.56 \mathrm{mg} / \mathrm{dl}$ \\
\hline $\mathrm{Na}$ & $142 \mathrm{mmol} / 1$ \\
\hline $\mathrm{K}$ & $4.0 \mathrm{mmol} / \mathrm{l}$ \\
\hline $\mathrm{Cl}$ & $104 \mathrm{mmol} / \mathrm{l}$ \\
\hline
\end{tabular}

WBC, white blood cell; Neutro, neutrophils; Lympho, lymphocyte; Mono, monocyte; Eosino, eosinophils; Baso, basophil; RBC, red blood cell; Hb, hemoglobin; Ht, hematocrit; PLT, platelet; Alb, serum albumin; TP, total protein; AST, aspartate aminotransferase; ALT, alanine aminotransferase; LDH, lactate dehydrogenase; ALP, alkaline phosphatase; $\gamma$-GTP, $\gamma$-glutamyl transpeptidase; $\mathrm{Cr}$, creatinine clearance; CRP, C-reactive protein; $\mathrm{Na}$, serum sodium; $\mathrm{K}$, serum potassium; $\mathrm{Cl}$, serum chlorine; $\mathrm{CMV}$, cytomegalovirus; $\mathrm{CD}$, Clostridium difficile.

History of the present illness: As part of chemoradiation therapy (CRT), the patient received two courses of cisplatin/vinorelbine combination therapy in November 2018. A total dose of $64 \mathrm{~Gy} / 40 \mathrm{Fr}$ was used for radical irradiation of the chest and mediastinum. Response assessment showed a stable disease. Durvalumab monotherapy was then initiated on day 44 after RT in January 2019 as a post-CRT maintenance therapy.

Treatment course: The clinical course after seven courses of durvalumab is shown in Fig. 3. The day on which the seventh course of durvalumab was introduced was set as day 0 . The patient was hospitalized for thorough examination and treatment on day 15 due to diarrhea (bloody stool) occurring 27 times/day (grade 3). Laboratory findings on admission are summarized in Table III. Colonoscopy revealed redness, erosion, edema, and grade 3 (Mayo classification) 

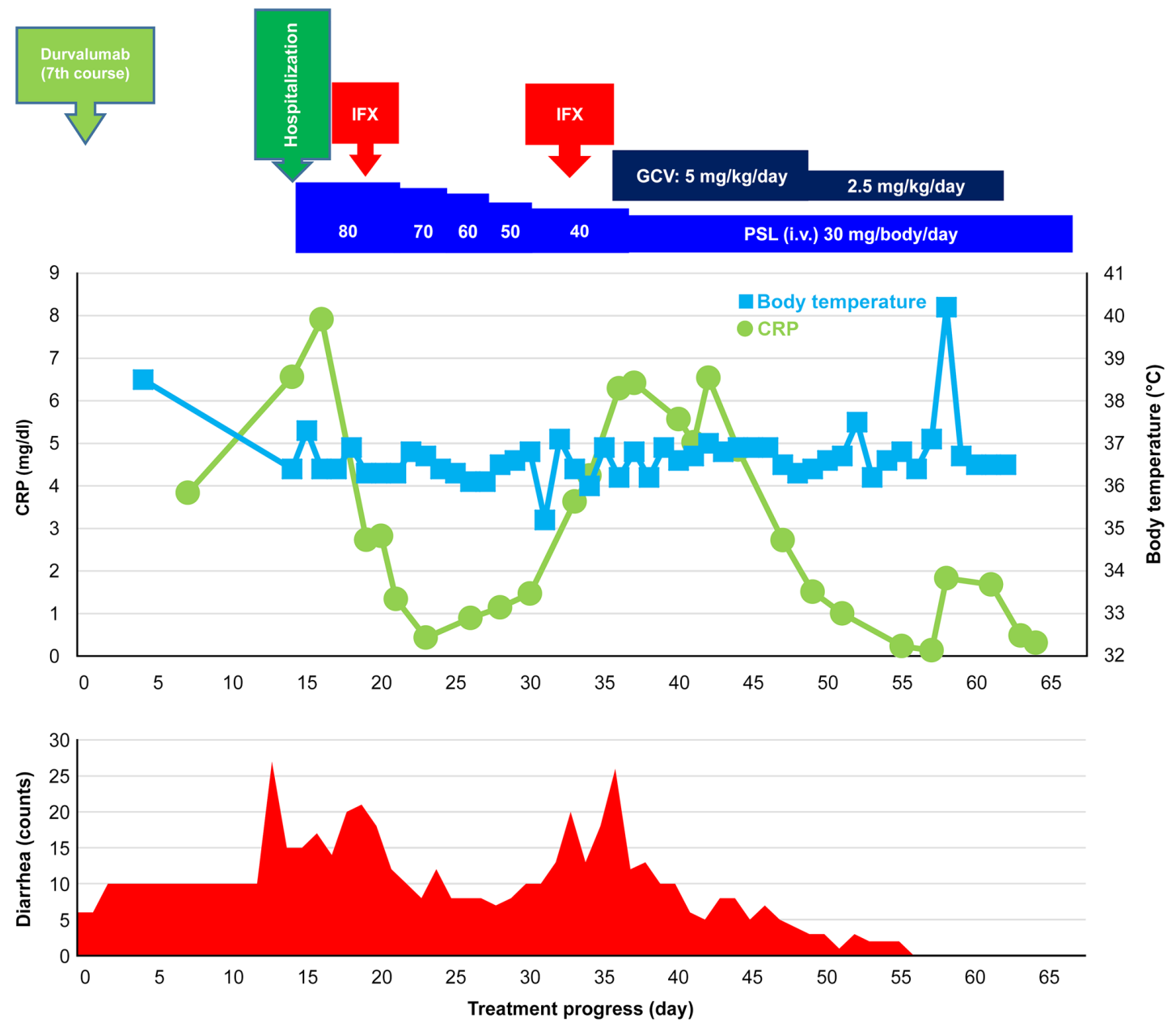

Figure 3. Clinical course after seven courses of durvalumab in case no. 2. IFX, infliximab; GCV, ganciclovir; PSL, prednisolone; CRP, C-reactive protein; i.v., intravenous.
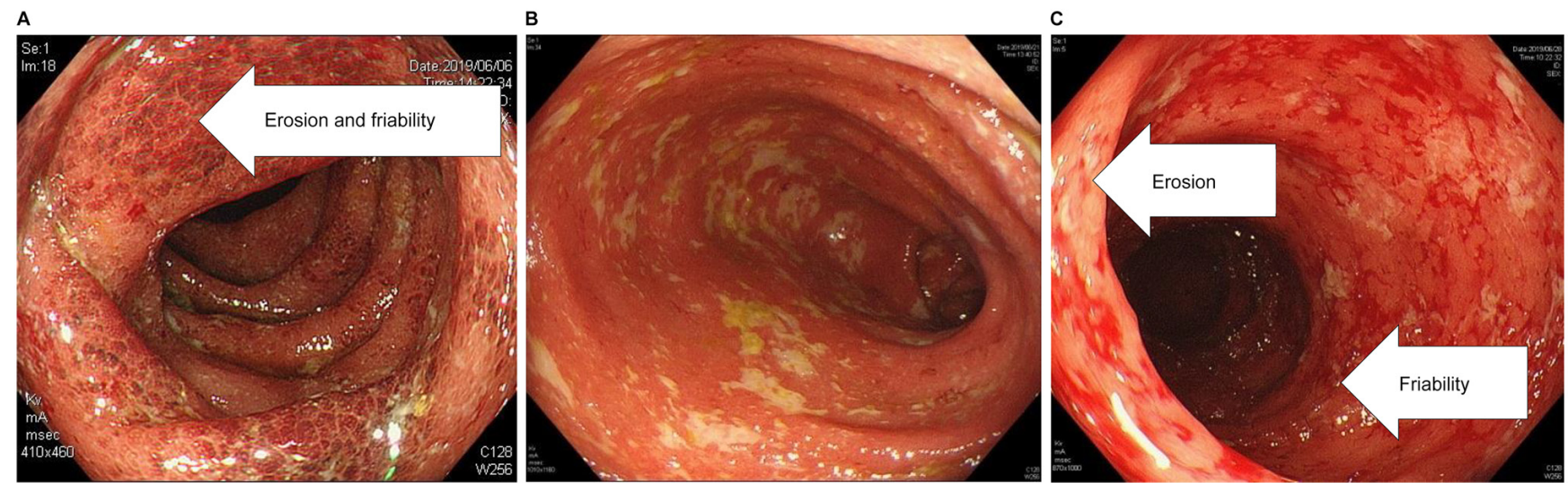

Figure 4. Lower gastrointestinal endoscopy in case no. 2. (A) Extensive erosion and friability were noted (day 15). (B) Erosion and friability improved transiently (day 30). (C) Erosion and friability worsened again (day 37).

lesions throughout the colon (Fig. 4), and computed tomography revealed wall thickening of the rectum, sigmoid colon, and cecum. Based on these findings, the patient was diagnosed with grade 3 colitis as an irAE. Treatment with high-calorie infusion, antibacterial cefmetazole infusion, and oral sulfamethoxazole/trimethoprim in conjunction with daily administration of PSL $1 \mathrm{mg} / \mathrm{kg} /$ day $(80 \mathrm{mg} /$ body/day) was initiated in a fasted state. On day 19, diarrhea occurred 21 times/day (grade 3), and the stool remained muddy. Thus, steroid treatment was concluded to be ineffective. Thereafter, IFX $5 \mathrm{mg} / \mathrm{kg} / \mathrm{day}$ ( $375 \mathrm{mg} /$ body/day) was administered with the steroid dose being gradually reduced, which improved his diarrhea to grade $1 / 2$. On day 30 , diarrhea remained persistent at 10 times/day (grade 2), and colonoscopy revealed 
improved albeit persistent mucosal erosion and bleeding. Thus, IFX re-administration was planned. On day 33, diarrhea worsened to 23 times/day (grade 3), during which he received the second dose of IFX $5 \mathrm{mg} / \mathrm{kg} /$ day ( $350 \mathrm{mg} /$ body/day). On day 36, no improvement in diarrhea frequency was observed (17 times/day; grade 3). While the CMV cell test result indicated CMV infection, the CD test result was negative. The patient was thus diagnosed with concomitant CMV enteritis and irAE (colitis) and was administered with ganciclovir $5 \mathrm{mg} / \mathrm{kg}$ infusion twice daily. On day 44, the CMV cell test result was negative, and diarrhea frequency improved to 9 times/day (grade 2). Ganciclovir dose was subsequently reduced to $2.5 \mathrm{mg} / \mathrm{kg} / \mathrm{dose} \mathrm{x} 2$ doses/day on day 50 . On day 51 , diarrhea was resolved (once/day; grade 0), and on day 69, treatment was switched to oral PSL $30 \mathrm{mg} /$ body/day. PSL treatment was continued thereafter with gradually tapering of doses, during which no diarrhea occurred.

\section{Discussion}

Various irAEs have been reported for Nivo and other ICIs considering their utility for the treatment of various cancers, including non-small cell lung cancer. In Japan, ICIs were first approved for malignant melanoma. Given the expected increase in opportunities for the use of ICIs, the incidence of grade $\geq 3$ diarrhea/colitis as a serious irAE has been predicted to increase accordingly. In particular, anti-CTLA-4 antibody preparations have been associated with a high incidence of severe diarrhea/colitis and possible poor outcomes, such as long-term hospitalization and death (13-15). In contrast, anti-PD-1 and anti-PD-L1 antibody preparations have been associated with relatively lower incidences of severe diarrhea/colitis (16-19).

Berman et al (20) reported that IPI-induced diarrhea/colitis can be attributable to the dysregulation of gastrointestinal mucosal immunity. Although the aforementioned study did not describe anti-PD-1 and anti-PD-L1 antibody preparations, these classes of ICIs presumably alter intestinal immunity in a manner similar to anti-CTLA-4 antibodies.

One study showed that tumor necrosis factor- $\alpha$ (TNF- $\alpha)$ was elevated in PD-1-knockout irAE model mice (21). While the anti-TNF- $\alpha$ antibody preparation IFX, a standard therapeutic agent for common ulcerative colitis, has been suggested as an effective treatment for irAEs, no specific IFX doses and dosing intervals have been recommended for this. Moreover, many reports have used IFX for enteritis in accordance with the treatment of ulcerative colitis (3-11). Pagès et al (6) proposed early administration of IFX 5-10 mg/kg/day when systemic steroid treatment failed to produce any appreciable symptomatic improvement $2 / 3$ days after the onset of IPI-induced irAEs. In the present study, seven patients with grade 3 diarrhea/colitis received a single or multiple doses of IFX $5.0 \mathrm{mg} / \mathrm{kg} /$ day according to the dosage and administration for ulcerative colitis. The median interval between systemic steroid administration and the first IFX dose was 9 (range, 2-39) days. However, we cannot exclude the possibility that such a long interval before initiating IFX administration affected treatment outcomes. Patients included in this study, who received multiple IFX doses, presented differing IFX dosing intervals given that the second and subsequent doses were provided according to relapse severity or prolonged symptoms. In the three responders to IFX, the effect appeared at a median of 18 (range, 9-32) days after IFX administration, which maintained diarrhea/colitis at grade 0 for at least 7 consecutive days. This finding suggests that IFX requires certain duration to produce its efficacy. Case nos. 6 and 7 experienced rapid deterioration of their general condition after IFX administration and ultimately died before IFX could show any beneficial effects. However, whether IFX administration had any causal relationship with the deaths remains unclear. Careful consideration may be warranted before IFX administration, particularly among patients showing rapid deterioration of the general condition.

Johnson et al (11) reported that severe diarrhea/colitis as an irAE improved in $72 \%$ (26/36) patients after a single dose of IFX, in $22 \%(8 / 36)$ after two doses, and in 6\% (2/36) after three doses. Furthermore, Soularue et al (1) pointed out that severe diarrhea/colitis as an irAE exhibits many clinical similarities with ulcerative colitis but rarely develops into chronic autoimmune diseases and that the former is likely to involve transient immune activation. Case nos. 4 and 5 in the present study received three or more doses of IFX and required long treatment periods for severe diarrhea/colitis (107 days and 86 days, respectively).

Other causes of grade $\geq 2$ diarrhea/colitis, including bacterial and viral enteritis, such as CMV and CD infections, must be ruled out (22). Accordingly, none of the eight patients developed any infection concurrent with irAEs. In case no. 2, however, neither systemic steroid treatment nor IFX was effective, with the patient ultimately being diagnosed with concomitant CMV enteritis based on reexamination results for infectious diseases. After immediate initiation of ganciclovir infusion, diarrhea improved to grade 0 after 14 days. Franklin et al (23) reported that $12.2 \%(5 / 41)$ patients with ICI-induced severe diarrhea/colitis were refractory to immunomodulatory treatment with steroids and IFX, showed more severe inflammation during colon biopsy, and tested positive for CMV. Kuo et al (24) detected CD in a case of severe diarrhea/colitis occurring after IPI administration in which symptoms improved transiently with steroids and IFX but relapsed after 1 month. While the mentioned reports do not discuss the mechanisms underlying CMV or CD infection, administration of the strong immunosuppressant IFX together with long-term systemic administration of high-dose steroids might have compromised the intestinal immunity, rendering the patient susceptible to opportunistic infections. In this study, patients in whom IFX exhibited its effects relatively earlier may be less susceptible to infection owing to the shorter treatment duration. The single dose of IFX used herein induced a full response only in case no. 1. In the remaining cases, CMV and CD infections were detected despite appreciable IFX effects and anti-CMV and antibacterial treatment for infections was accordingly administered in parallel. These data suggest that periodic assessment for CMV and CD infections is necessary when IFX is administered.

In addition, the immunosuppressant cyclosporine (CyA) and vedolizumab, a humanized $\alpha 4 \beta 7$ integrin monoclonal antibody, are worth considering as third-line treatments for severe diarrhea/colitis as irAE. Accordingly, Iyoda et al (25) reported 
that oral CyA $50 \mathrm{mg} /$ body/day as a third-line treatment for Nivo-induced grade 3 enteritis, following the unsuccessful first- and second-line treatments with oral PSL 30-60 mg for 50 days and two doses of IFX $5 \mathrm{mg} / \mathrm{kg}$, respectively, decreased the frequency of diarrhea after 3 days and resolved diarrhea after 2 weeks. Moreover, Bergqvist et al (26) reported that vedolizumab promoted remission in 6 of the 7 patients with malignant melanoma or lung cancer who developed IPI- or Nivo-induced enteritis refractory to steroids and IFX at a median of 56 days after administration with no related AEs.

According to Postow et al (27) it is possible that immunosuppression with IFX, steroids, and other agents reduce the antitumor efficacy of ICIs. They compared the antitumor efficacy of ICIs in patients who received immunosuppressants for the treatment of irAE with that in those who did not, and they found no significant reduction between the two groups; however, they did not eliminate the possibility of reduction because no prospective studies have been conducted. Nevertheless, using immunosuppressants for irAE treatment has been reported to increase the likelihood of contracting opportunistic infections, as was the case in this study.

The use of IFX for irAE treatment is not covered by national health insurance, the guideline on optimal usage issued by the Ministry of Health, Labour and Welfare of Japan states that 'when corticosteroids do not improve adverse reactions, the addition of immunosuppressants other than corticosteroids should be considered,' thereby officially recommending the use of immunosuppressants as needed. Accordingly, attending physicians administered the immunosuppressants recommended for patient's irAE according to the ASCO or other guidelines. We retrospectively included and analyzed cases in which IFX was administered at discretion of individual attending physicians, with no new interventions. Therefore, we categorized our study as a retrospective observational study.

Limitations of this study were its retrospective design based on electronic medical records and a small number of patients as it was a single-center study, which predisposes it to various biases due to insufficient statistical power. Therefore, a multi-center study involving a larger number of patients is necessary for more accurate assessments.

In conclusion, early initiation of IFX treatment in conjunction with systemic steroid therapy should be considered for severe diarrhea/colitis and other irAEs. However, reevaluation for possible infections and prompt revision of the treatment strategy, such as switching to oral CyA or vedolizumab, may be necessary when irAEs do not respond to steroids/IFX.

\section{Acknowledgements}

Not applicable.

\section{Funding}

No funding was received.

\section{Availability of data and materials}

The datasets used and/or analyzed during the current study are available from the corresponding author on reasonable request.

\section{Authors' contributions}

Through research conferences, MT, TY, KF, TIn, SO, YN, SY, TO, RI, TIs, TK, KN and FI established comprehensive research goals and aims, and developed and designed methodologies. TIn, SO, YN, SY, TO, RI, TIs, TK and KN provided patient samples. YK, MT, TY, KF, TIn, SO, YN, SY, TO, RI, TIs, TK, KN and FI performed experiments, collected data and conducted administrative activities to maintain the survey data. YK and AT conducted data analysis. YK drafted the manuscript with help from AT. TIn, SO, YN, SY, TO and KN assessed the authenticity of all the raw data to ensure its legitimacy. MT, TY, KF, TIn, SO, YN, TO, SY, RI, TIs, TK, KN and FI conducted critical reviews, visualizations and edits. AT, KF and FI were responsible for oversight and leadership in planning and carrying out research activities. All authors agreed to be accountable for all aspects of the work in ensuring that questions related to the accuracy or integrity of any part of the work are appropriately investigated and resolved. All authors read and approved the final manuscript.

\section{Ethics approval and consent to participate}

The present study was conducted with approval from the Ethical Review Board of the Osaka International Cancer Institute (Osaka, Japan) and in accordance with ethical guidelines on clinical research (approval no. 19086). Due consideration was given toward protecting personal information, with data being handled after anonymization. Information on the present study is available at the institution's website. The patients could withdraw their consent for participation at any period throughout the study.

\section{Patient consent for publication}

Not applicable.

\section{Competing interests}

The authors declare that they have no competing interests.

\section{References}

1. Soularue E, Lepage P, Colombel JF, Coutzac C, Faleck D, Marthey L, Collins M, Chaput N, Robert C and Carbonnel F: Enterocolitis due to immune checkpoint inhibitors: A systematic review. Gut 67: 2056-2067, 2018.

2. Brahmer JR, Lacchetti C, Schneider BJ, Atkins MB, Brassil KJ, Caterino JM, Chau I, Ernstoff MS, Gardner JM, Ginex P, et al: Management of immune-related adverse events in patients treated with immune checkpoint inhibitor therapy. J Clin Oncol 36: 1714-1768, 2018.

3. Minor DR, Chin K and Kashani-Sabet M: Infliximab in the treatment of anti-CTLA4 antibody (Ipilimumab) induced immune-related colitis. Cancer Biother Radiopharm 24: 321-325, 200

4. Beck KE, Blansfield JA, Tran KQ, Feldman AL, Hughes MS, Royal RE, Kammula US, Topalian SL, Sherry RM, Kleiner D, et al: Enterocolitis in patients with cancer after antibody blockade of cytotoxic T-lymphocyte-associated antigen 4 . J Clin Oncol 24: 2283-2289, 2006.

5. Hillock NT, Heard S, Kichenadasse G, Hill CL and Andrews J: Infliximab for ipilimumab-induced colitis: A series of 13 patients Asia Pac J Clin Oncol 13: e284-e290, 2017.

6. Pagès C, Gornet JM, Monsel G, Allez M, Bertheau P, Bagot M, Lebbé $C$ and Viguier M: Ipilimumab-Induced acute severe colitis treated by infliximab. Melanoma Res 23: 227-230, 2013. 
7. Geier M, Gouva S and Geier B: Enterocolitis due to nivolumab treated by infliximab. ASGIS 2: 14-16, 2019.

8. Yanai S, Nakamura S and Matsumoto T: Nivolumab-induced colitis treated by infliximab. Clin Gastroenterol Hepatol 15: e80-e81, 2017.

9. Nassri AB, Muenyi V, Al-Khasawneh A, De Souza Ribeiro B, Scolapio JS, Malespin M and de Melo SW Jr: Ipilimumab and nivolumab induced steroid-refractory colitis treated with infliximab: A case report. World J Gastroint Pharmacol Ther 10: 29-24, 2019.

10. Delasos L, Desai A, Lia NL, Kethireddy N and Ray C: A case of immunotherapy-induced colitis complicated by perforation and treated with infliximab postoperatively. Case Rep Oncol Med 2019: 9069354, 2019.

11. Johnson DH, Zobniw CM, Trinh VA, Ma J, Bassett RL Jr, Abdel-Wahab N, Anderson J, Davis JE, Joseph J, Uemura M, et al: Infliximab associated with faster symptom resolution compared with corticosteroids alone for the management of immune-related enterocolitis. J Immunother Cancer 6: 103, 2018.

12. Perez-Ruiz E, Minute L, Otano I, Alvarez M, Ochoa MC, Belsue V, de Andrea C, Rodriguez-Ruiz ME, Perez-Gracia JL, Marquez-Rodas I, et al: Prophylactic TNF blockade uncouples efficacy and toxicity in dual CTLA-4 and PD-1 immunotherapy. Nature 569: 428-432, 2019.

13. Hodi FS, O'Day SJ, McDermott DF, Weber RW, Sosman JA, Haanen JB, Gonzalez R, Robert C, Schadendorf D, Hassel JC, et al: Improved survival with ipilimumab in patients with metastatic melanoma. N Engl J Med 363: 711-723, 2010.

14. Robert C, Thomas L, Bondarenko I, O'Day S, Weber J, Garbe C, Lebbe C, Baurain JF, Testori A, Grobb JJ, et al: Ipilimumab plus dacarbazine for previously untreated metastatic melanoma. N Engl J Med 364: 2517-2526, 2011.

15. Gupta A, De Felice KM, Loftus EV Jr and Khanna S: Systematic review: Colitis associated with anti-CTLA-4 therapy. Aliment Pharmacol Ther 42: 406-417, 2015.

16. Robert C, Ribas A, Wolchok JD, Hodi FS, Hamid O, Kefford R, Weber JS, Joshua AM, Hwu WJ, Gangadhar TC, et al: Anti-P rogrammed-Death-Receptor-1 treatment with pembrolizumab in Ipilimumab-refractory advanced melanoma: A randomised dose-comparison cohort of a phase 1 trial. Lancet 384: 1109-1117, 2014.

17. Hamid O, Robert C, Daud A, Hodi FS, Hwu WJ, Kefford R, Wolchok JD, Hersey P, Joseph RW, Weber JS, et al: Safety and tumor responses with lambrolizumab (anti-PD-1) in melanoma. N Engl J Med 369: 134-144, 2013.

18. Weber JS, D'Angelo SP, Minor D, Hodi FS, Gutzmer R, Neyns B, Hoeller C, Khushalani NI, Miller WH Jr, Lao CD, et al: Nivolumab versus chemotherapy in patients with advanced melanoma who progressed after anti-CTLA-4 treatment (CheckMate 037): A randomised, controlled, open-label, phase 3 trial. Lancet Oncol 16: 375-384, 2015.
19. Rizvi NA, Mazières J, Planchard D, Stinchcombe TE, Dy GK, Antonia SJ, Horn L, Lena H, Minenza E, Mennecier B, et al: Activity and safety of nivolumab, an anti-PD-1 immune checkpoint inhibitor, for patients with advanced, refractory squamous non-small-cell lung cancer (CheckMate 063): A phase 2, single-arm trial. Lancet Oncol 16: 257-265, 2015.

20. Berman D, Parker SM, Siegel J, Chasalow SD, Weber J, Galbraith S, Targan SR and Wang HL: Blockade of cytotoxic T-lymphocyte antigen- 4 by ipilimumab results in dysregulation of gastrointestinal immunity in patients with advanced melanoma. Cancer Immun 24: 10-11, 2010.

21. Liu J, Blake SJ, Harjunpää H, Fairfax KA, Yong MC, Allen S, Kohrt HE, Takeda K, Smyth MJ and Teng MW: Assessing immune-related adverse events of efficacious combination immunotherapies in preclinical models of cancer. Cancer Res 76: 5288-5301, 2016.

22. Weber JS, Kähler KC and Hauschild A: Management of immune-related adverse events and kinetics of response with ipilimumab. J Clin Oncol 30: 2691-2697, 2012.

23. Franklin C, Rooms I, Fiedler M, Reis H, Milsch L, Herz S, Livingstone E, Zimmer L, Schmid KW, Dittmer U, et al: Cytomegalovirus reactivation in patients with refractory checkpoint inhibitor-induced colitis. Eur J Cancer 86: 248-256, 2017.

24. Kuo JR, Davis AD, Rodriguez EA, Vela MF, Heigh RI, Salomao MA and Gurudu SR: Severe diarrhea in the setting of immune checkpoint inhibitors. Case Rep Gastroenterol 12: 704-708, 2018

25. Iyoda T, Kurita N, Takada A, Watanabe H and Ando M: Resolution of infliximab-refractory nivolumab-induced acute severe enterocolitis after cyclosporine treatment in a patient with non-small cell lung cancer. Am J Case Rep 27: 360-364, 2018.

26. Bergqvist V, Hertervig E, Gedeon P, Kopljar M, Griph H, Kinhult S, Carneiro A and Marsal J: Vedolizumab treatment for immune checkpoint inhibitor-induced enterocolitis. Cancer Immunol Immunother 66: 581-592, 2017.

27. Postow MA, Sidlow R and Hellmann MD: Immune-Related adverse events associated with immune checkpoint blockade. N Engl J Med 378: 158-168, 2018.

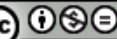

This work is licensed under a Creative Commons Attribution-NonCommercial-NoDerivatives 4.0 International (CC BY-NC-ND 4.0) License. 